POS PROCEEDINGS

\title{
Vertex and track reconstruction in ATLAS and CMS
}

Jiří Mašík* on behalf of the ATLAS and CMS collaborations

University of Manchester

E-mail: Jiri.Masikemanchester.ac.uk

The performance of the ATLAS and CMS tracking detectors is discussed paying special attention to those parameters that are of interest for heavy-flavour physics - transverse impact parameters and primary vertex resolution.

14th International Conference on B-Physics at Hadron Machines April 8-12, 2013

Bologna, Italy

${ }^{*}$ Speaker. 


\section{Introduction}

The performance of tracking and vertexing plays a crucial role in B-physics and heavy flavour physics in general. A precise reconstruction of tracks and vertices has a direct impact on b-tagging and on precision measurements, for example lifetime measurements. A high reconstruction efficiency is important for a full reconstruction of b-quark decays and for identification of signal and background events. A short introduction of the ATLAS and CMS tracking detectors and of the software for reconstruction of tracks and primary vertices is presented here together with detailed performance measurements.

\section{The tracking detectors of ATLAS and of CMS}

The ATLAS Inner Detector [1] is shown in Figure 1(a). It combines silicon and gas subdetectors. Closest to the beam line is the Pixel detector, followed by the double-sided silicon-strip detector (SCT) and the transition radiation tracker (TRT). The physical characteristics of the subdetectors are given in Table 1. The Inner Detector is surrounded by a $2 \mathrm{~T}$ solenoidal magnet.

The CMS tracker [2] is based solely on silicon detectors and provides tracking information within a similar volume to ATLAS. It is segmented into barrel and endcap parts and consists of two subsystems: the pixel detector and the strip tracker. The strip tracker combines layers of double-sided and of single-sided modules. The layout of the tracker is shown in Figure 1(b) with parameters listed in Table 2. The solenoidal magnetic field in which it is immersed is $3.8 \mathrm{~T}$. A similar $\mathrm{R} \phi$ resolution as in ATLAS is achieved with larger pixel dimensions thanks to position interpolation with an analogue read-out.

In both experiments the track reconstruction starts from seeds in the inner layers of the silicon detectors and a combinatorial Kalman filter is used to add more hits. In the case of CMS this procedure is iterative and seeds with looser constraints are taken into account in the following iterations.

Primary vertices are reconstructed in two steps, first is the iterative vertex finding, where clusters of tracks with compatible z-positions along the beamline form vertex candidates followed by the vertex fit where vertex positions and their uncertainties are determined. The details of the reconstruction techniques are discussed elsewhere $[3,4,5]$.

\begin{tabular}{|c|c|c|c|c|}
\hline Subdetector & Radius $[\mathrm{cm}]$ & Sensor size $[\mu \mathrm{m}]$ & Resolution $[\mu \mathrm{m}]$ & $<$ Hits on track> \\
\hline \hline Pixel & $5-12.3$ & $50 \times 400$ & $\mathrm{R} \phi: 10 \mathrm{z}: 115$ & 3 \\
\hline SCT & $30-51$ & 80 & $\mathrm{R} \phi: 16 \mathrm{z}: 580$ & 8 \\
\hline TRT & $56-108$ & $\mathrm{R}=2 \mathrm{~mm}$ & 130 & 30 \\
\hline
\end{tabular}

Table 1: Parameters of the ATLAS Inner Detector

\begin{tabular}{|c|c|c|c|c|}
\hline Subdetector & Radius $[\mathrm{cm}]$ & Sensor size $[\mu \mathrm{m}]$ & Resolution $[\mu \mathrm{m}]$ & $<$ Hits on track> \\
\hline \hline Pixel & $4.4-10.2$ & $100 \times 150$ & $\mathrm{R} \phi: 10 \mathrm{z:20}$ & 3 \\
\hline Strip tracker & $25.5-110$ & $\sim 100$ & $\sim 15-\sim 45$ & 13 \\
\hline
\end{tabular}

Table 2: Parameters of the CMS tracker 


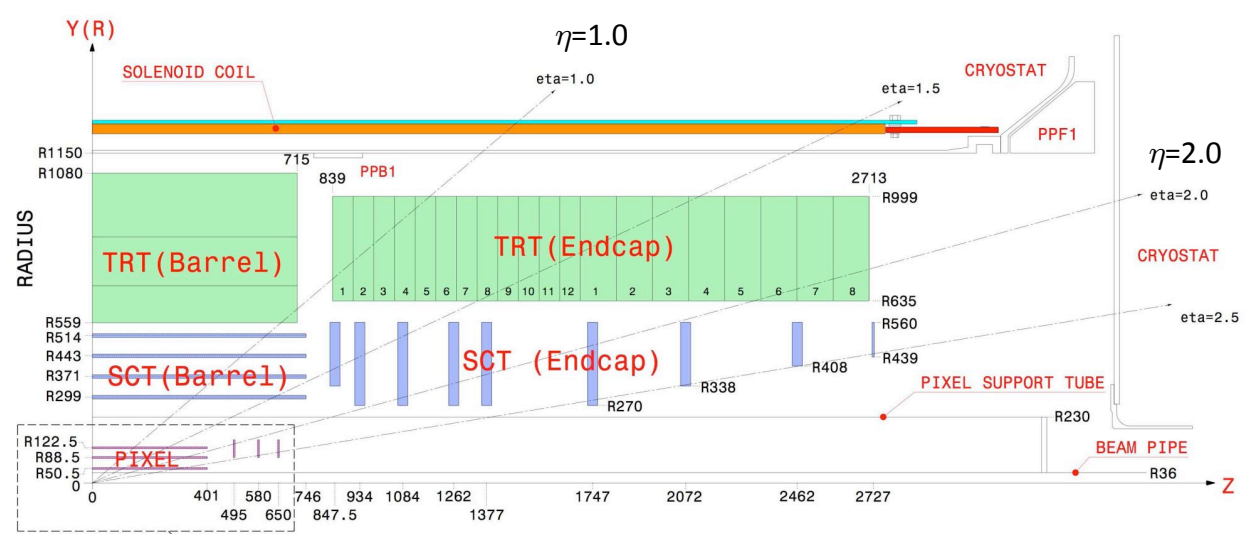

(a)

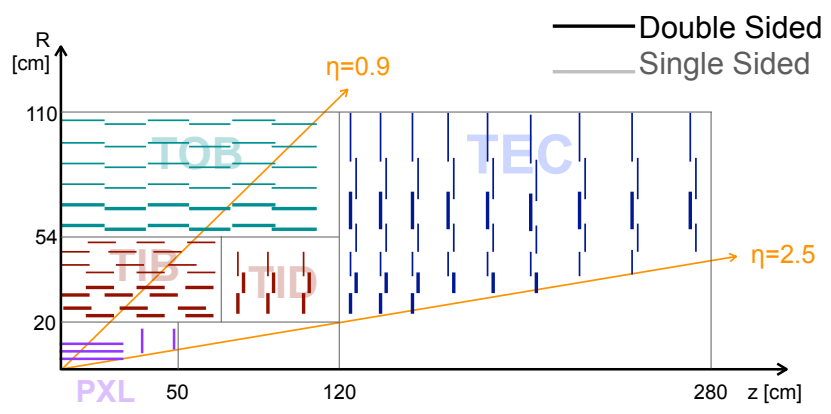

(b)

Figure 1: The layout of the (a) ATLAS inner detector and of the (b) CMS tracker.

\section{The performance of the tracking and vertexing}

The performance of the vertexing has been studied by a data driven method referred to as the split-vertex technique, where tracks forming a vertex are split into two sets and vertices are fit independently to each. From a difference in their reconstructed position a vertex position resolution is derived. The resolutions of the vertex position in the transverse $(x)$ and longitudinal $(z)$ coordinate are shown as a function of the number of tracks in the vertex in Figure 2. The resolutions in the transverse coordinate converge to a similar value $20 \mu \mathrm{m}$ for minimum bias events both for ATLAS and CMS respectively. The longitudinal resolution reaches $30 \mu \mathrm{m}$ and $20 \mu \mathrm{m}$ for ATLAS and CMS respectively. In addition the ATLAS result shows resolutions from the minimum bias Monte Carlo demonstrating a very good understanding of the detector performance. The CMS result also shows a comparison of the resolutions in minimum bias data with a jet enriched data sample where smaller resolutions are achieved due to higher transverse momentum spectrum of the tracks.

The resolution of the transverse impact parameter, $d_{0}$, corrected for the spread of the primary interaction point, is shown in Figure 3(a) for ATLAS and Figure 3(b) for CMS. The $d_{0}$ resolution in ATLAS is presented as a function of $1 / \sqrt{p^{2} \sin ^{3}(\theta)}$ factorizing the effect of multiple scattering for tracks in the pseudorapidity range $0<\eta<0.25$. The $d_{0}$ resolution in CMS is shown as a function of the track pseudorapidity in 3 bins of track transverse momentum. The resolution degrades with lower track momentum and with the amount of traversed material towards higher pseudorapidity 


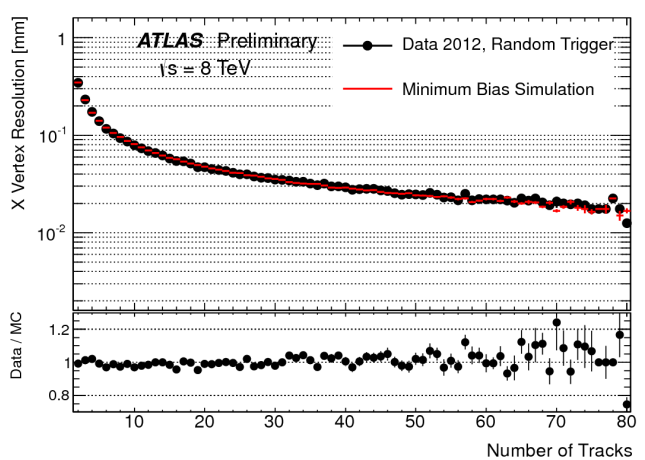

(a)

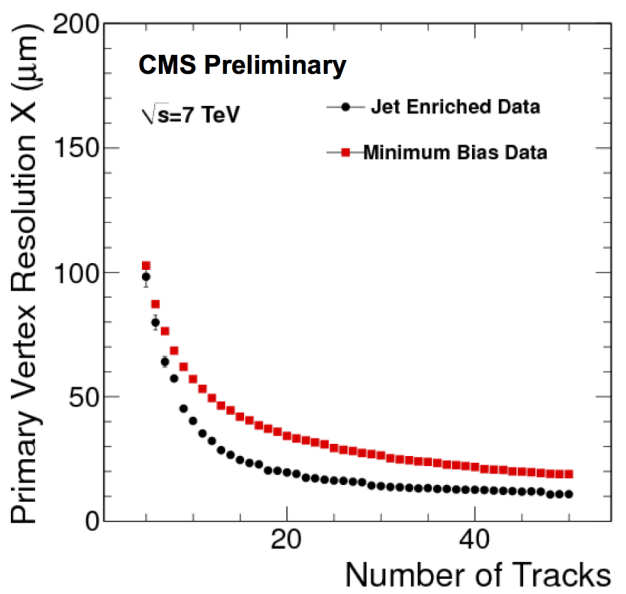

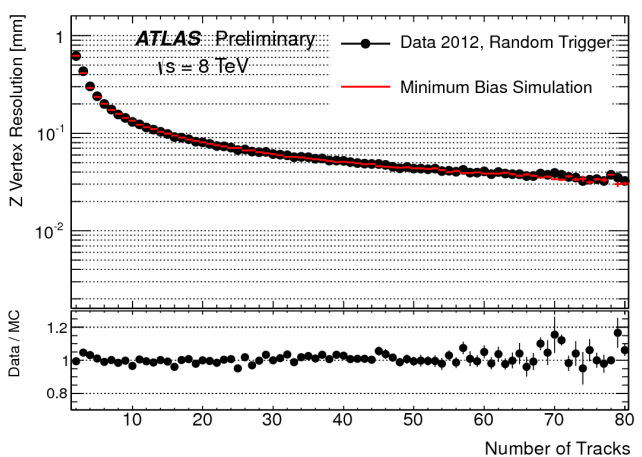

(b)

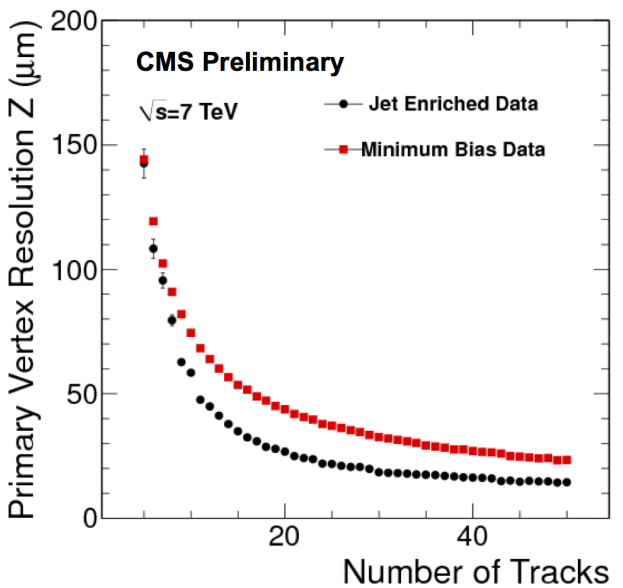

(c)

Figure 2: Vertex position resolutions in one transverse (left) and in longitudinal coordinate (right) for ATLAS (top) and CMS (bottom) [6, 7].

as a consequence of multiple scattering. The resolution in the central region of the detector ranges from $10 \mu \mathrm{m}$ and $100 \mu \mathrm{m}$ and is similar for both the ATLAS and CMS detectors. The resolutions are compared with MC simulations and a very good agreement has been achieved in both experiments.

The robustness of the tracking and vertexing with increased detector occupancies due to the presence of multiple interactions is one of the concerns of increasing the luminosity in the LHC. Increased occupancy may cause an increased number of fake tracks and vertices and a degradation of the efficiencies and resolutions. In ATLAS the number of reconstructed tracks as a function of the number of reconstructed vertices was studied. It is shown in Figure 4(a) for two reconstruction settings, the default, and the robust. The robust configuration has an increased requirement on the number of hits per track and reduces the number of possible missing hits on tracks in active detector modules. For the default setting the number of reconstructed tracks increases faster with the vertex multiplicity indicating an increased rate of fake tracks, the linear behaviour is restored with the robust configuration. The effect of tightening the track quality cuts on the tracking efficiency is small. The robust settings were used also in the Figure 4(b) where normalized distributions of the number of tracks as a function of the transverse impact parameter are compared for events with 


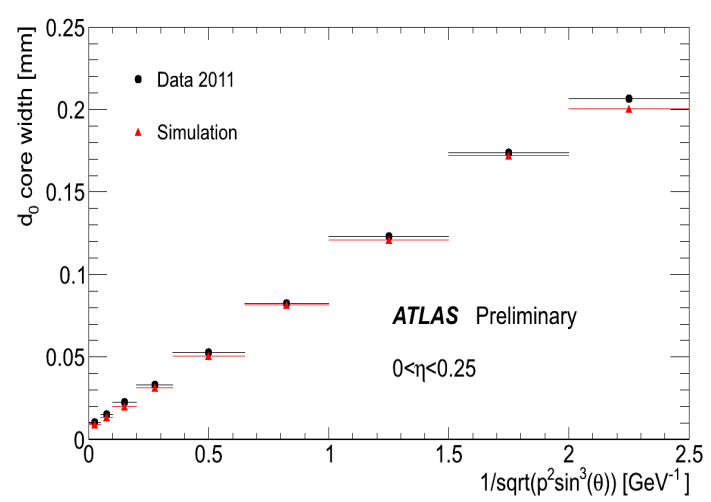

(a)

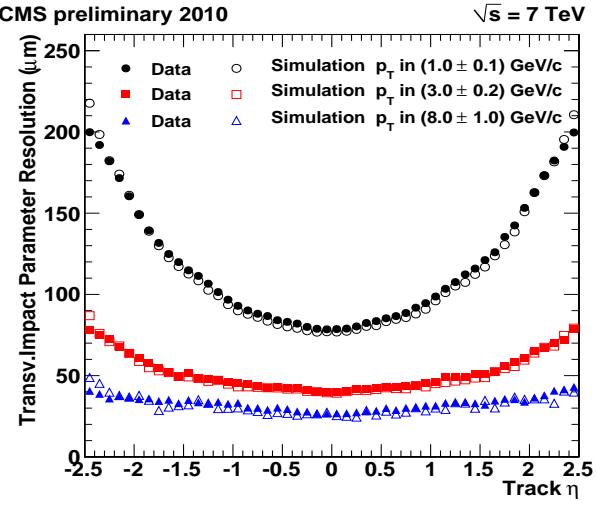

(b)

Figure 3: The resolution of the transverse impact parameter of tracks as a function of the track pseudorapidity and transverse momentum for ATLAS (left) and CMS (right) [6, 5].

three different levels of pile-up. The fact that the tails of the distributions do not differ indicate stable performance in terms of both efficiency and fake rate for tracks with larger $d_{0}$.

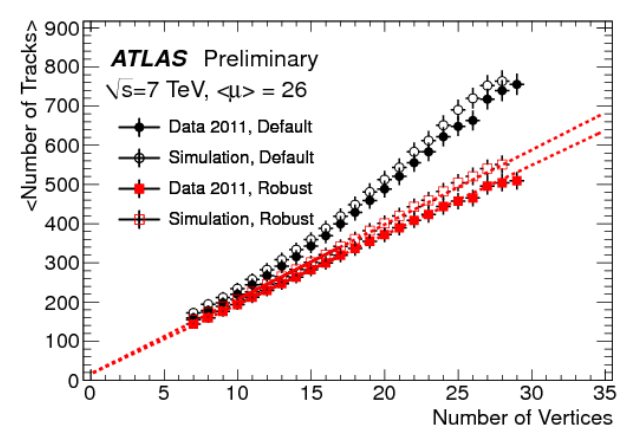

(a)

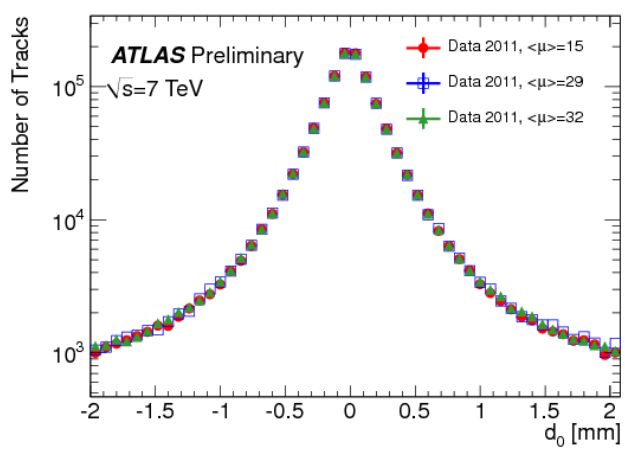

(b)

Figure 4: Mean number of reconstructed tracks as a function of the number number of vertices using the default and pile-up robust settings (a). Distribution of the number of tracks as a function of the transverse impact parameter $d_{0}$ for the ATLAS track reconstruction with the pile-up robust settings (b) [3].

Another study of pile-up related effects was done with CMS High-Level Trigger. Primary vertices in the trigger of CMS are reconstructed using data from the pixel detector as a fast alternative to the full reconstruction. The number of pixel vertices reconstructed from pixel tracks is shown in Figure 5(a) as a function of the nominal number of interactions computed from the instantaneous luminosity and the inelastic cross section. The expected linear increase is observed, in this case with data from two fills over the range of 15 to 31 interactions per event.

\section{Summary}

The B-physics and heavy-flavour program of the ATLAS and CMS experiments depends upon the excellent performance of their tracking detectors and related reconstruction software. Precision 


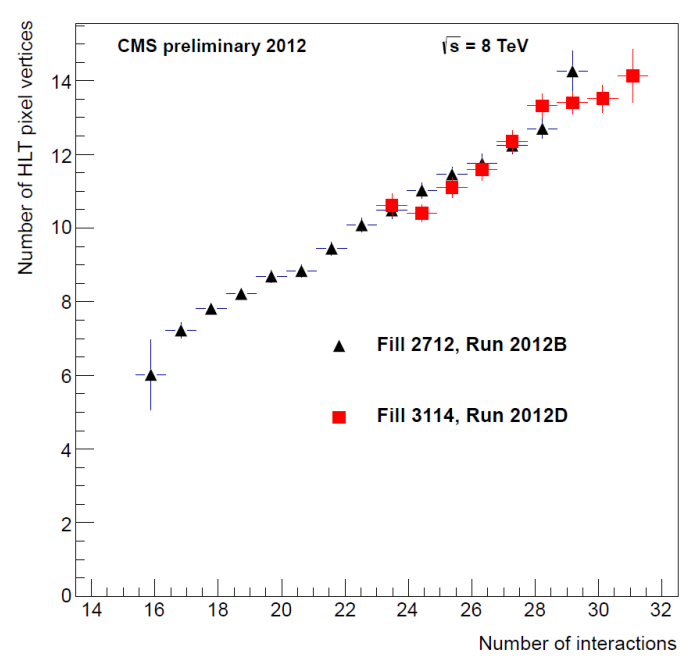

(a)

Figure 5: The number of the pixel vertices reconstructed by the high-level trigger of CMS as a function of the nominal number of interactions [7].

track and vertex reconstruction has been demonstrated in the vertex position and transverse impact parameter resolutions. The design of the detectors and the track and vertex reconstruction is robust with respect to detector occupancies caused by pile-up interactions which in 2012 have already exceeded design limits for the first stage of the LHC operation.

\section{References}

[1] ATLAS Collaboration, The ATLAS Experiment at the CERN Large Hadron Collider, JINST 3 (2008) S08003.

[2] The CMS Collaboration, The CMS experiment at the CERN LHC, JINST 3 (2008) S08004.

[3] The ATLAS Collaboration, Performance of the ATLAS Inner Detector Track and Vertex Reconstruction in the High Pile-Up LHC Environment, ATLAS-CONF-2012-042, https://cds.cern.ch/record/1435196

[4] T. Cornelissen et al., Concepts, Design and Implementation of the ATLAS New Tracking (NEWT), ATL-SOFT-PUB-2007-007, https://cds.cern.ch/record/1020106

[5] The CMS collaboration, Tracking and Primary Vertexing Results in First 7 TeV collisions, CMS-PAS-TRK-10-005, https://cds.cern.ch/record/1279383

[6] ATLAS public plots https://twiki.cern.ch/twiki/bin/view/AtlasPublic/InDetTrackingPerformanceApprovedPlots http://atlas.web.cern.ch/Atlas/GROUPS/PHYSICS/IDTRACKING/PublicPlots/ATL-COM-PHYS2012-474 http://atlas.web.cern.ch/Atlas/GROUPS/PHYSICS/IDTRACKING/PublicPlots/ATL-COM-PHYS2012-471

[7] CMS Public plots https://twiki.cern.ch/twiki/bin/view/CMSPublic/PhysicsResultsTRK 